\title{
Preface by the Editors
}

\author{
"The problem of two bodies on Einstein's theory remains an outstanding \\ challenge to mathematicians - like the problem of three bodies on Newton's \\ theory."
}

Arthur Eddington, The Mathematical theory of Relativity [Sec. 44, p. 95]

\begin{abstract}
During the dawn of General Relativity (GR), the formidable mathematical complexity of Einstein's theory made clear that the use of approximation (analytical) methods was mandatory, in order to tackle many problems of physical interest. Einstein himself pioneered both post-Newtonian and linearized approximations to investigate planetary motion and gravitational waves. Beyond the scope of these methods, the extreme regime of gravity — strong field with strong dynamics would remain completely unexplored, largely due to the absence of any (astro) physical hints of its relevance, in the real universe.

A turn of events took place in the last half century. The discovery of quasars and pulsars, the growing evidence for black holes and gravitational waves, motivated a theoretical understanding of extreme gravity. Simultaneously, the advent of scientific computing brought in a new ally to assist in the struggle to tame extremely complicated equations. The dawn of numerical relativity (NR) took place in the 1960s, providing physicists and mathematicians with a new powerful tool to address the outstanding challenge observed by Eddington.
\end{abstract}

The following decades witnessed slow progress, hampered by numerous technical, conceptual and implementational difficulties. Success in addressing Eddington's challenge appeared only in the early 21st century, with the renowned 2005 breakthroughs in evolving black hole binaries. Ten years later, this century long effort was beautifully vindicated by the direct detection of (at least) two gravitational wave events (GW150914 and GW151226) interpreted as sourced by the inspiral and merger of black hole binaries, an interpretation for which NR waveforms were central.

In parallel, the powerful computational infrastructure that was developed to address Eddington's challenge, became useful to investigate other problems, beyond GR or even beyond astrophysics, often motivated by high energy physics (HEP). Many of these problems were discussed at an exploratory "Numerical Relativity and 
High Energy Physics" (NR/HEP) meeting, held at the island of Madeira, in 2011, that led to a community effort to summarize outstanding problems in this field of research (Class. Quantum Grav. 29 (2012) 244001). As a further effort, a NR/HEP network was approved by the European Union, within the Marie Curie's International Research Staff Exchange Scheme (IRSES) programme, (NRHEP-295189FP7-PEOPLE-2011-IRSES), coordinated by the six of us. From January 2011 to December 2015, this network promoted research in this interface, multidisciplinary field, catalyzing some of the most prominent scientific results in the area, gathering the international community in various meetings and pushing the production of reference reviews in the topic (e.g. Living Rev. Rel. 18 (2015) 1 and Class. Quantum Grav. 32 (2015) 243001). The last such meeting took place in Belém, Brazil, in September-October 2015, coinciding with the celebration of the centennial of GR and with the Third Amazonian Symposium on Physics. This volume is a selection of the contributions to this meeting, a tip of the iceberg of the exciting recent developments within Einstein's theory and its generalizations, in particular using numerical techniques.

Carlos A. R. Herdeiro

Departamento de Fúsica da Universidade de Aveiro and Center for Research in Mathematics and Applications (CIDMA), Campus de Santiago, 3810-183 Aveiro, Portugal

Emanuele Berti

Department of Physics and Astronomy, The University of Mississippi University, MS 3867\%, USA

CENTRA, Departamento de Física, Instituto Superior Técnico, Universidade de Lisboa, Avenida Rovisco Pais 1, 1049 Lisboa, Portugal

Vitor Cardoso,

Department of Physics and Astronomy, The University of Mississippi University, MS 38677, USA

CENTRA, Departamento de Física, Instituto Superior Técnico, Universidade de Lisboa, Avenida Rovisco Pais 1, 1049 Lisboa, Portugal

Perimeter Institute for Theoretical Physics, 31 Caroline Street North Waterloo, Ontario, Canada N2L $2 Y 5$

Faculdade de Física, Universidade Federal do Pará, 66075-110, Belém, Pará, Brazil

Dipartimento di Fisica,

"Sapienza" Università di Roma $\mathcal{G}$ Sezione INFN Roma1, P.A. Moro 5, 00185, Roma, Italy 
Luis C. B. Crispino Faculdade de Física, Universidade Federal do Pará, 66075-110, Belém, Pará, Brazil

Leonardo Gualtieri Dipartimento di Fisica, "Sapienza" Università di Roma \& Sezione INFN Roma1, P.A. Moro 5, 00185, Roma, Italy

Ulrich Sperhake Department of Physics and Astronomy, The University of Mississippi University, MS 38677, USA Department of Applied Mathematics and Theoretical Physics, University of Cambridge, Cambridge CB3 0WA, UK California Institute of Technology, Pasadena, CA 91125, USA

June 2016 